\title{
Cardiovascular magnetic resonance assessment in previously repaired ALCAPA
}

Aurelio Secinaro*, Hopewell Ntsinjana, Vivek Muthurangu, Oliver Tann, Marina L Hughes, Victor Tsang,

Andrew M Taylor

From 2011 SCMR/Euro CMR Joint Scientific Sessions

Nice, France. 3-6 February 2011

\section{Introduction}

Anomalous origin of the left coronary artery from the pulmonary artery (ALCAPA) is a rare coronary artery anomaly.

Purpose: The aim of this study is to show the role of cardiovascular magnetic resonance (CMR) in assessing these patients.

\section{Methods}

6 patients with repaired ALCAPA (2 Tackeuchi, 4 direct re-implantation) underwent CMR to assess clinical suspicion of myocardial ischemia - short axis and long axis cine images (assess ventricular function), late-gadolinium enhancement (detect myocardial fibrosis), adeno- sine stress perfusion scan (detection of reversible ischaemia) and 3D whole-heart imaging (visualization of proximal coronary arteries).

\section{Results}

The LV function was preserved in all patients (mean $\mathrm{LVEF}=62.7 \% \pm 4.23 \%$ ). The LV volumes were within the normal ranges LV (mean indexed LVEDV $=75.4 \mathrm{ml}$ \pm 3.5 , mean $\mathrm{LVESV}=31.6 \mathrm{ml} \pm 9.37)$ In one patient, hypokinesia of the anterior segments was visualized. 5 of the 6 patients showed sub-endocardial late gadolinium enhancement involving the antero-lateral wall and the anterior papillary muscle. 4 of the 6 patients presented areas of inducible ischemia, reversible at rest. In 3 of the

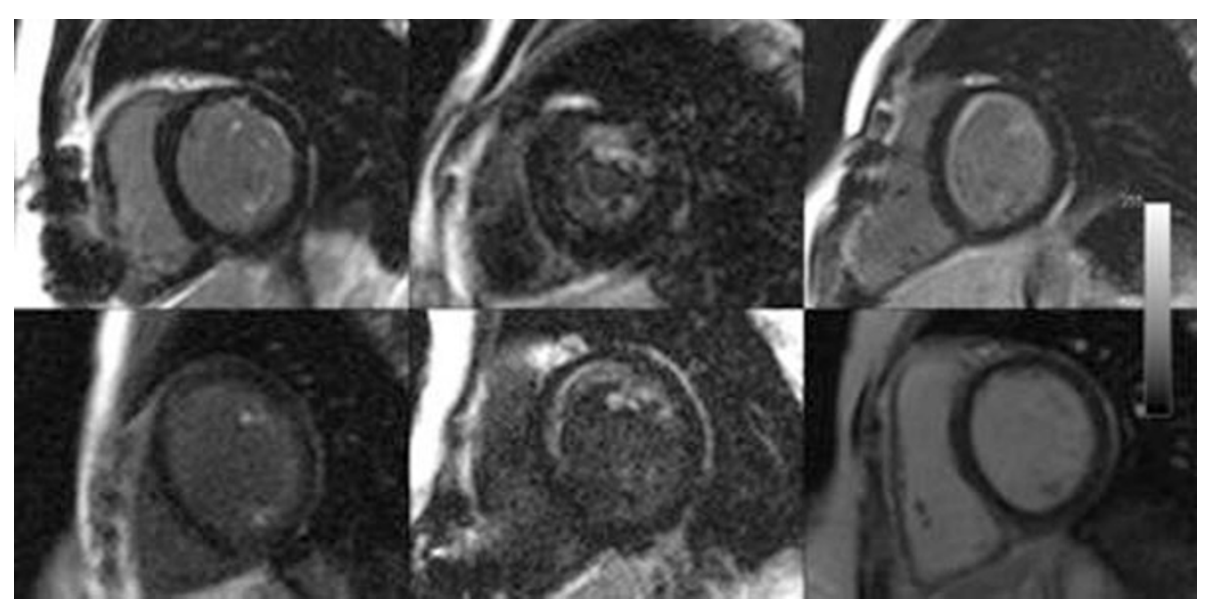

Figure 1 The charachteristic LGE in our repaired ALCAPA population. 


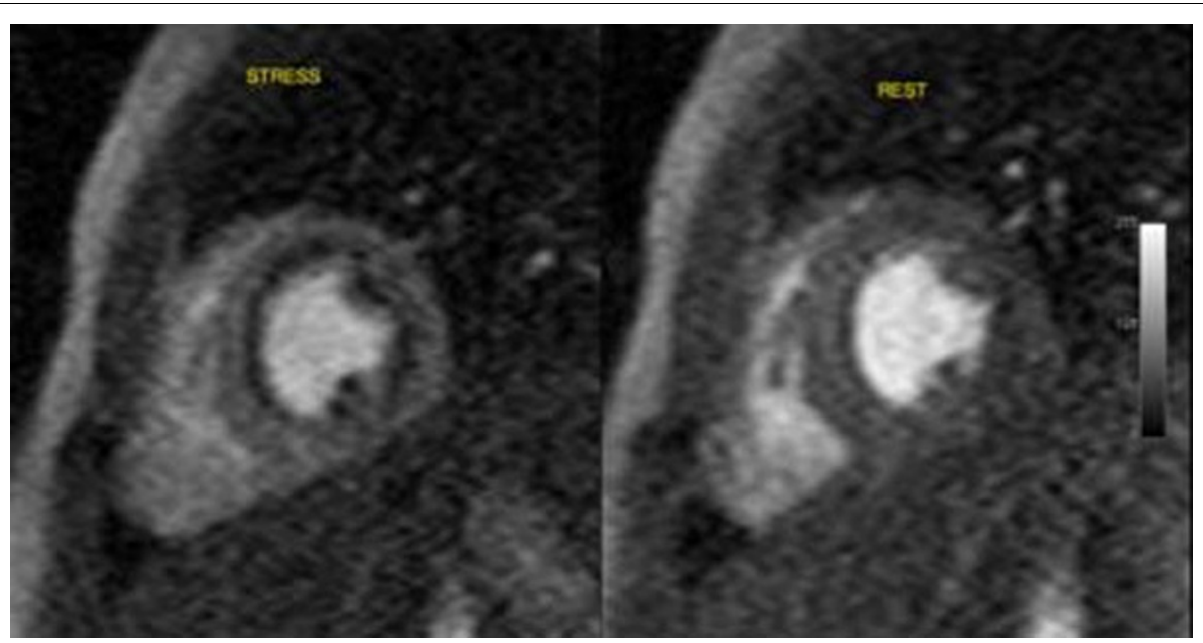

Figure 2 Perfusion defect in repaired ALCAPA.

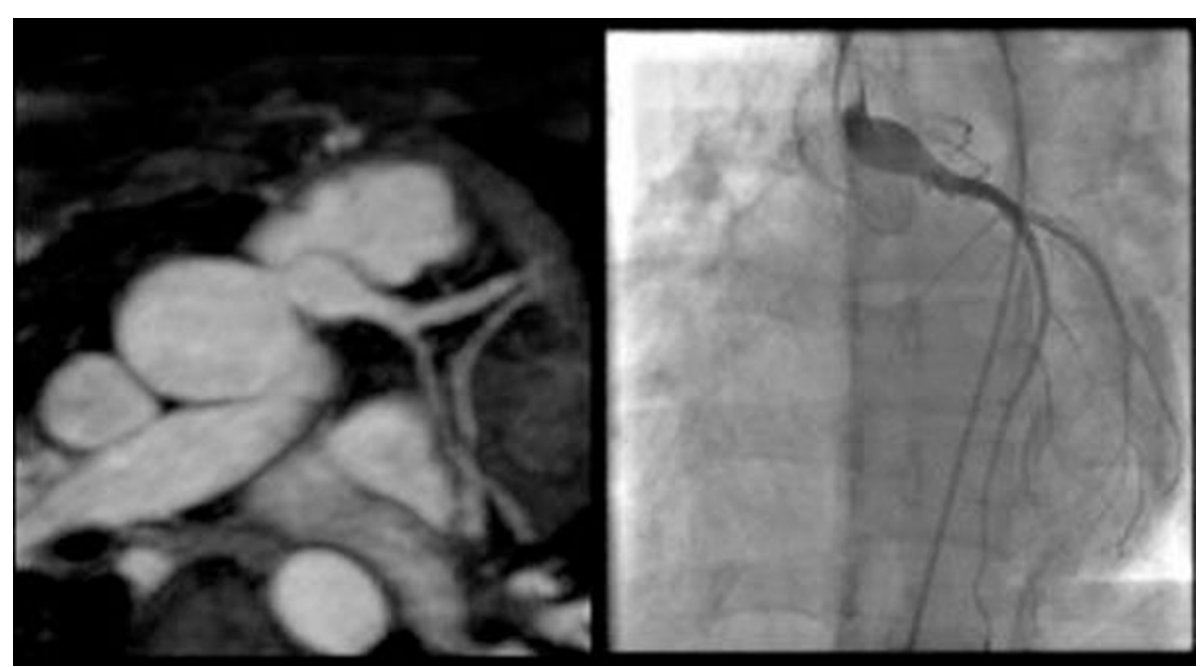

Figure 3 3D whole-heart imaging of ALCAPA repair compared with invasive coronary angiography(Tackeuchi technique).

6 patients the proximal course of the left coronary artery wasn't clearly visualized. In these patients, proximal the LCA obstruction was confirmed during invasive coronary angiography.

\section{Conclusions}

CMR is a good, non-invasive, radiation-free investigation in the post-surgical evaluation of ALCAPA. We show that antero-lateral sub-endocardial myocardial fibrosis is a characteristic finding. Furthermore, stress adensoine CMR perfusion, can identify reversible ischaemia in this group. Such imaging may be a promising tool in the future clinical decision -making.

\section{doi:10.1186/1532-429X-13-S1-P192}

Cite this article as: Secinaro et al:: Cardiovascular magnetic resonance assessment in previously repaired ALCAPA. Journal of Cardiovascular Magnetic Resonance 2011 13(Suppl 1):P192. 